\title{
Effect of strong magnetic field on the first-order electroweak phase transition
}

\author{
R. Fiore, ${ }^{*}$ A. Tiesi ${ }^{\dagger}$ \\ Dipartimento di Fisica, Università della Calabria, \\ 87036 Arcavacata di Rende, Cosenza, Italy \\ L. Masperi $\$$ A. Mégevand $\$$ \\ Centro Atómico Bariloche and Instituto Balseiro, \\ Comisión Nacional de Energía Atómica and Universidad Nacional de Cuyo, \\ 8400 San Carlos de Bariloche, Argentina
}

\begin{abstract}
The broken-symmetry electroweak vacuum is destabilized in the presence of a magnetic field stronger than a critical value. Such magnetic field may be generated in the phase transition and restore the symmetry inside the bubbles. A numerical calculation indicates that the first-order phase transition is delayed but may be completed for a sufficient low value of the Higgs mass unless the magnetic field is extremely high.
\end{abstract}

\section{Introduction}

It has been found that very strong magnetic fields are capable of destabilizing the electroweak vacuum by forming a vector boson $W^{+} W^{-}$condensate and restoring the symmetry [1]. The required field can only be thought to have existed at the very beginning of the universe and one of the possibilities is that it was generated during the electroweak phase transition [2, 3]. This primordial field may have been subsequently the seed of the present galactic magnetic field [4].

One may wonder whether the restoration of symmetry caused by this strong magnetic field can delay the electroweak phase transition. In particular, if it is of first-order the magnetic field might avoid its completion through the bubble mechanism.

The simplest way to see why a strong magnetic field can destabilize the electroweak vacuum is to consider the energy of a charged spin-one particle interacting with a uniform magnetic field along the 3 -axis

$$
E_{N}^{2}=p_{3}^{2}+m_{0}^{2}+(2 N+1) e B-g e B
$$

* fiore@fermi.fis.unical.it

$\dagger$ tiesi@fermi.fis.unical.it

$\ddagger$ masperi@cab.cnea.edu.ar

$\S$ megevand@cab.cnea.edu.ar

ฯ Fellow of Consejo Nacional de Investigaciones Científicas y Técnicas, Argentina. 
For the lowest Landau level $N=0$ if the gyromagnetic factor $g$ is 2 as occurs in the $W$ case, it is clear that the effective mass will become zero for

$$
B_{c}=\frac{m_{W}^{2}}{e} \simeq 10^{24} G
$$

This expression is analogous to that of the critical electric field required to create pairs through tunneling.

If one wishes to calculate the decay probability of the vacuum, one must evaluate

$$
Z=<0\left|e^{-i H t}\right| 0>=e^{-i t\left(E_{v a c}-i \frac{\Gamma}{2}\right)} .
$$

In Euclidean metric the one-loop amplitude for a scalar field depends on $\operatorname{det}\left(-D_{E}^{2}+m^{2}\right)$ with $D_{E \mu}=\partial_{\mu}-i e A_{\mu}$, being $D_{E 4}=i D_{0}$. Using the Schwinger proper time method [5] one obtains

$$
\ln Z=\int_{0}^{\infty} \frac{d s}{s} \operatorname{tr} e^{-\left(-D_{E}^{2}+m^{2}\right) s} .
$$

For constant electromagnetic fields the trace is known to give the vacuum energy density [6]

$$
\rho=-\int_{0}^{\infty} \frac{d s}{s} \frac{e^{-m^{2} s}}{(4 \pi s)^{2}}\left[\frac{e s \sqrt{E^{2}-B^{2}}}{\sin \left(e s \sqrt{E^{2}-B^{2}}\right)}-1\right],
$$

where the -1 comes from subtracting $\rho(A=0)$. This integral has a logarithmic divergence for $s=0$ which can be absorbed renormalizing fields and charge [5].

In Eq.(5) for $E>B$ the integral has poles in the $s$-axis which give origin to an imaginary part corresponding to pair creation. We will be instead interested in the case of $E=0$ and constant magnetic field for which

$$
\rho=-\int_{0}^{\infty} \frac{d s}{s} \frac{e^{-m^{2} s}}{(4 \pi s)^{2}}\left[\frac{e s B}{\sinh (e s B)}-1\right]
$$

that has no poles.

For the spin- $1 W_{\mu}$ case we adopt the view that the only modification to $\ln Z$ is the interaction of spin with magnetic field $2 e \mathbf{B} \cdot \mathbf{s}$ in the exponent of Eq.(田). Now the trace must be performed on momentum and spin states where the latter involves this added interaction to give

$$
\ln Z=\int_{0}^{\infty} \frac{d s}{s}\left(e^{-2 e B s}+e^{2 e B s}+1\right) \operatorname{tr} e^{-\left(-D_{E}^{2}+m_{W}^{2}\right) s} .
$$

Since the remaining trace is equal to the scalar case, the relevant part of the vacuum energy density is

$$
\rho=-\int_{0}^{\infty} \frac{d s}{s} \frac{e^{-m_{W}^{2} s}}{(4 \pi s)^{2}}\left[\frac{e s B}{\sinh (e s B)} 2 \cosh (2 e B s)\right] .
$$

This expression has no poles but diverges for $s \rightarrow \infty$ when $B>B_{c}=m_{W}^{2} / e$ due to the gyromagnetic factor 2 of the $W$ boson, which would not occur either for $g=1$ or for the $s=1 / 2$ case. This divergence is an indication of the vacuum instability for large magnetic field. The decay rate should be evaluated in the more realistic situation of $B$ increasing with time, with the consequent generation of an electric field.

In our calculation of the next section we will not take into account the evolution with time of the magnetic field but we will consider that when its value is larger than the critical one in the region of a bubble containing the broken-symmetry vacuum, the bubble will be destroyed. 


\section{Delay of the phase transition due to magnetic field}

It is known that the phase transition of the standard model is of first order only for Higgs masses which are below the experimental bound [7]. However we will not attempt to use an extension as the MSSM to allow consistency with this bound since our goal is to establish the effect of a strong magnetic field on the first-order transition with a reasonably simple effective potential. In the minimal standard model this temperature dependent potential for the Higgs field $\varphi$ is 8

$$
V(\varphi, T)=D\left(T^{2}-T_{0}^{2}\right)|\varphi|^{2}-E T|\varphi|^{3}+\frac{\lambda_{T}}{4}|\varphi|^{4}
$$

where $D=\frac{1}{8 v^{2}}\left(2 m_{W}^{2}+m_{Z}^{2}+2 m_{t}^{2}\right) \quad, \quad E=\frac{1}{6 \pi v^{2}}\left(2 m_{W}^{3}+m_{Z}^{3}\right) \quad$,

$\lambda_{T}=\lambda-\frac{3}{16 \pi^{2} v^{4}}\left(2 m_{W}^{4} \ln \frac{m_{W}^{2}}{a_{B} T^{2}}+m_{Z}^{4} \ln \frac{m_{Z}^{2}}{a_{B} T^{2}}-4 m_{t}^{2} \ln \frac{m_{t}^{2}}{a_{F} T^{2}}\right) \quad, \quad T_{0}^{2}=\frac{1}{4 D}\left(m_{H}^{2}-\frac{8 B}{v^{2}}\right) \quad$, $B=\frac{3}{64 \pi^{2}}\left(2 m_{W}^{4}+m_{Z}^{4}-4 m_{t}^{4}\right) \quad, \quad \ln a_{B}=3.91 \quad, \quad \ln a_{F}=1.14$.

All the parameters of the potential Eq.(9) are determined by the experimental masses of gauge bosons and top quark, $m_{W}=80 \mathrm{GeV}, m_{Z}=91 \mathrm{GeV}, m_{t}=175 \mathrm{GeV}$ and the breaking scale $v=246 \mathrm{GeV}$, with the only unknown given by the Higgs mass $m_{H}$.

The first-order transition will occur in the range from $T_{c}=\left[\frac{T_{0}^{2}}{1-E^{2} /\left(\lambda_{T_{c}} D\right)}\right]^{1 / 2}$ to $T_{0}$, where $T_{c}$ corresponds to equal minima and $T_{0}$ to the disappearance of the barrier between them. In this range of temperatures bubbles of broken-symmetry phase are formed with a probability per unit volume and time given by the semiclassical approximation

$$
P(T) \simeq \omega_{f}^{4} e^{-E_{c} / T},
$$

where $\omega_{f}^{2}=\left.\frac{\partial^{2}}{\partial \varphi^{2}} V(\varphi, T)\right|_{\varphi=0} . E_{c}$ will come from the maximization of the bubble energy which, in the thin wall approximation of width $\varepsilon$, is

$$
E=-\frac{4 \pi}{3} R^{3} \Delta V+4 \pi R^{2} \gamma
$$

where the surface tension is

$$
\gamma \simeq \frac{(\Delta \varphi)^{2}}{\varepsilon}
$$

$\Delta V$ and $\Delta \varphi$ are the differences of potential and Higgs field between the two phases respectively. The maximization of Eq.(11) gives the critical radius $R_{c}$ and energy $E_{c}$

$$
R_{c}=\frac{2 \gamma}{\Delta V} \quad, \quad E_{c}=\frac{16 \pi}{3} \frac{\gamma^{3}}{(\Delta V)^{2}}
$$

The velocity of expansion of the bubble can be estimated by [9]

$$
v_{W}(T) \simeq \frac{\Delta V}{T^{4}}
$$

and the wall width by $\varepsilon \simeq m_{H}(T)^{-1}$ where $m_{H}\left(T_{c}\right) \simeq m_{H} / 50$, for not too high values of $m_{H}$.

We will consider that the phase transition will be completed when the average distance between centres of bubbles $2 R_{n}(t)$ is equal to twice their average radius $R_{b}(t)$, i.e. when 
$d(t)=2\left[R_{n}(t)-R_{b}(t)\right]$ vanishes (Alternatively, one could determine the moment at which the fraction of volume occupied by bubbles of new phase equals one [9, 10]). The relation between the time $t$ and temperature in the radiation regime is taken as

$$
t=k / T^{2} \quad, \quad k \sim 10^{16} \mathrm{GeV},
$$

to give the beginning of the phase transition at $t \sim 10^{-12} \mathrm{sec}$.

In terms of the probability for bubble formation, its number density will be

$$
n(t)=\int_{t_{c}}^{t} d t^{\prime} P\left(t^{\prime}\right)
$$

so that the average radius of the volume per bubble is

$$
R_{n}(T)=\left[\frac{4}{3} \pi n(T)\right]^{-1 / 3} .
$$

The average bubble radius comes from

$$
R_{b}(t)=\frac{1}{n(t)} \int_{t_{c}}^{t} R\left(t^{\prime}, t\right) P\left(t^{\prime}\right) d t^{\prime},
$$

where $R\left(t^{\prime}, t\right)=R_{c}\left(t^{\prime}\right)+v_{W}\left(t^{\prime}\right)\left(t-t^{\prime}\right)$.

Now we must include the influence of the magnetic field generated during the expansion of bubbles. Several mechanisms of creation of seed fields (e.g. charge separation in bubble walls, bubble collisions or fluctuating Higgs gradients) [2, 3] involve the appearance of non-zero expectation values of the Higgs field. This means that the seed fields will arise in general in the regions where bubbles are present. Once a seed is formed, the magnetic field is quickly amplified due to magnetic turbulence in the conducting fluid, until it reaches in this region the value given by energy equipartition. Therefore we will consider that when a bubble is produced a magnetic field

$$
B \simeq c \frac{T^{2}}{(v R)^{1 / 2}}
$$

will manifest itself in a region $R$ after a time $\tau$. The exponent of the denominator is the statistically most favorable one to have sizeable homogeneous fields as the region increases. The constant $c$ has a maximum value of 100 to satisfy the constraint given by nucleosynthesis 111. We will assume $\tau \simeq R_{c} / v_{W}$ which is in reasonable agreement with some mechanisms of magnetic field generation [2]. Since for $B \gtrsim B_{c}$ the decay rate of the electroweak vacuum, $\Gamma \sim\left(e B_{c}\right)^{2} \mathbb{1}$, is much larger than the bubble nucleation or expansion rates, we can consider that the bubble disappears because the symmetry inside it is restored. In this way $R\left(t^{\prime}, t\right)=0$ for $t^{\prime}+\tau<t$ and $B>B_{c}=m_{W}^{2} / e$ in (18) and the completion of the phase transition according to a bubble mechanism will be either delayed or avoided.

Our numerical calculation shows that for large values of $m_{H}$, e.g. $200 G e V$, the first-order phase transition is not completed even without magnetic field (see Fig.1).

For a low value of the Higgs mass $m_{H} \simeq 70 \mathrm{GeV}$ our model allows the completion of the first-order transition without magnetic field (see Fig.2). If the maximum possible value of the 


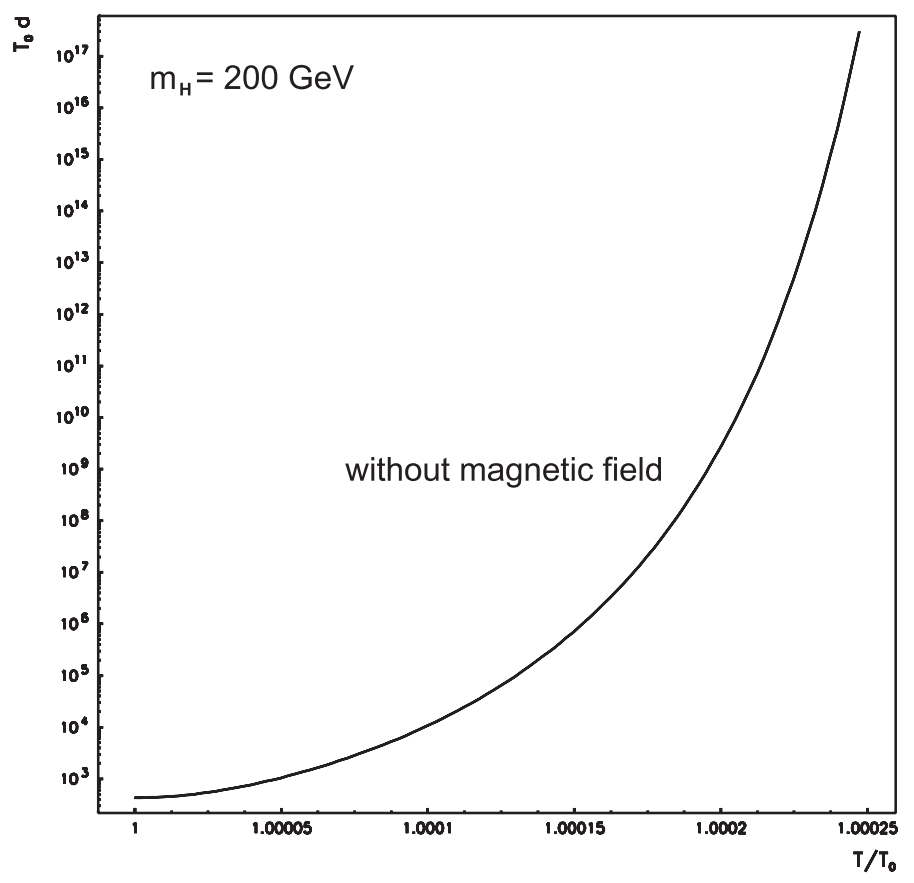

Figure 1: Mean distance between the walls of two bubbles in units of $1 / T_{0}$ versus temperature in units of $T_{0}$ for $m_{H}=200 \mathrm{GeV}$.

magnetic field $c=100$ is assumed, the first-order transition is avoided (Fig.2a). With a smaller value of the magnetic field, e.g. $c=36$, the completion of the first-order transition is only delayed in around $100 \mathrm{MeV}$ (Fig.2b), and well below this intensity the effect of the magnetic field is negligible.

An alternative scenario would be to consider that the magnetic field was generated in a prior stage of the universe, e.g. the GUT epoch. Its effect on the electroweak phase transition can be studied through an effective potential depending on temperature and constant magnetic field [12]. It is interesting that again for $m_{H} \lesssim 80 \mathrm{GeV}$ the magnetic field delays the first-order transition due to an increase of the free energy of the broken-symmetry phase. This result agrees with the tendency shown by our calculation but we remark that the two situations are different. In our case we do not have a magnetic field prior to the phase transition but a strong field is formed in small regions around the bubbles, destroying them. The magnetic field in a subhorizon volume will have a statistical average several orders of magnitude below the critical value, therefore its influence on the effective potencial will be negligible. If the first-order transition is not completed, at temperature $T_{0}$ our system will be at the maximum of the potential $\varphi \sim 0$ whereas the minimum will be at $\varphi \neq 0$ without any barrier. Now the phase transition will occur through the global rolling down of the field $\varphi$ towards the minimum of the potential.

\section{Conclusions}

We have seen that the highest possible magnetic field together with the most favorable law for having homogeneous field in regions of increasing size might have cosmological consequences 


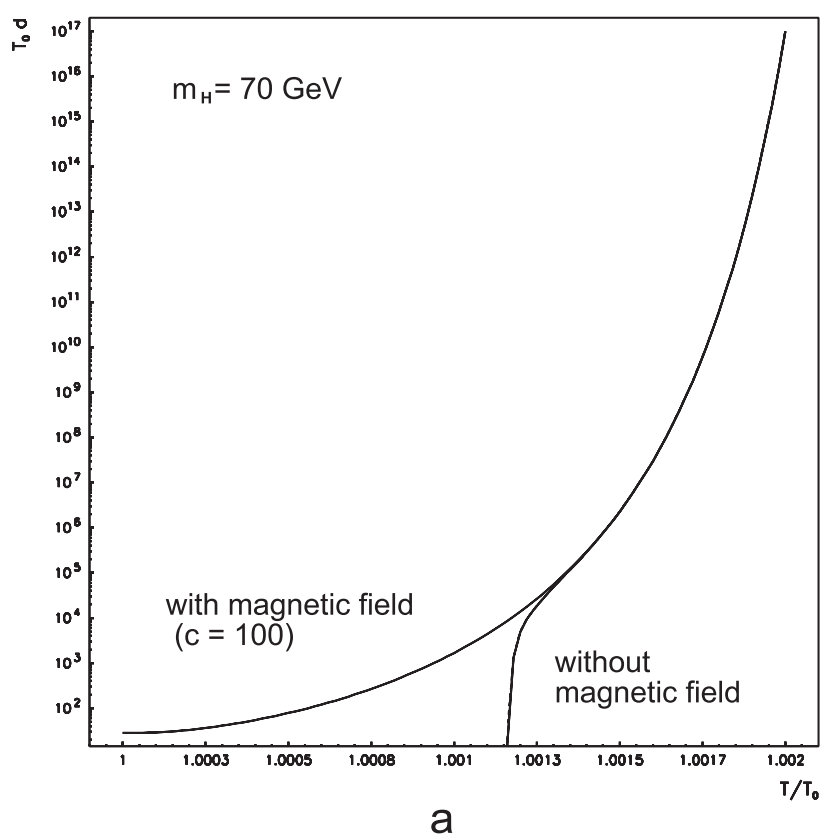

a

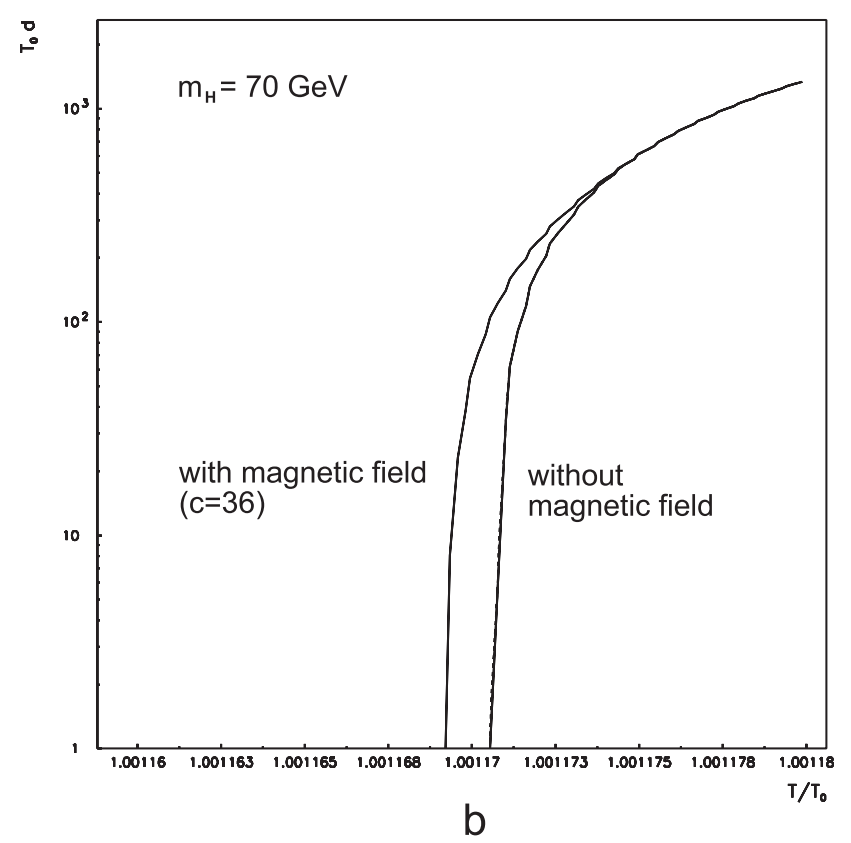

b

Figure 2: Mean distance between the walls of two bubbles in units of $1 / T_{0}$ versus temperature in units of $T_{0}$ for $m_{H}=200 \mathrm{GeV}$. a. High magnetic field $(c=100)$. b. Low magnetic field $(c=36)$.

through the non-completion of the first-order electroweak transition through a bubble mechanism. Therefore the usual electroweak baryogenesis due to bubble expansion would be affected. But one must notice that also the homogeneous increase of $\varphi$ can produce a matter-antimatter asymmetry. This is because there will be a baryonic chemical potential related to the time variation of the $C P$ violating phase $\theta$. The resulting baryonic density will depend on the variation $\Delta \theta$ in the interval when the sphalerons are active due to the smallness of $\varphi$. For a weakly first-order transition, an advantage of this mechanism compared to the bubble one is that the baryonic density would not be erased in the broken phase because here the value of $\varphi$ is larger due to the delay of the phase transition. One may remind that this problem is also avoided by the baryogenesis in cosmic strings but paying the price of a suppression factor in the active volume.

However, it is unlikely that such a strong and large size primordial magnetic field has occurred, and for more acceptable fields the effect would be only a small decrease of the temperature for the completion of a first-order transition.

We have studied the influence of the magnetic field on the phase transition using the easiest model, i.e. the standard model and not the MSSM where presumably the first-order phase transition can occur for not too light Higgs mass [13]. The fact that we obtain the completion of the first-order transition without magnetic field for $m_{H} \simeq 70 \mathrm{GeV}$, not far below the experimental bound is probably due to the definition that it occurs when the bubbles touch each other without taking into account their scattering. But we believe that the general conclusions on the magnitude of the effect do not depend on the details of the used electroweak model.

Regarding further developments of this calculation, it would be important to evaluate the vacuum decay rate caused by a time dependent magnetic field in order to consider more carefully 
its effect on the bubbles instead of taking the simplification of assuming their disappearance as soon as the magnetic field is larger than the critical value.

\section{Acknowledgments}

We are deeply indebted to A. Della Selva and R. Iengo for very stimulating discussions. L. M. and A. M. thank the kind hospitality of the Departments of Physics of the Universities of Napoli and della Calabria at Cosenza where parts of this research has been performed.

This work was partially supported by CONICET through PICT 0358.

\section{References}

[1] J. Ambjorn and P. Olesen, NBI-HE-93-17 (hep-ph/9304220); Int. Journ. Mod. Phys. A5 (1990) 4525; Nucl. Phys. B330 (1990) 625; Phys. Lett. 218B (1989) 67; Nucl. Phys. B315 (1989) 606.

[2] G. Baym, D. Bödeker and L. Mc Lerran, Phys. Rev. D53 (1996) 662.

[3] B. Cheng and A. V. Olinto, Phys. Rev. D50 (1994) 2451; G. Sigl, A. V. Olinto and K. Jedamzik, Phys.Rev. D55 (1997) 4582; T. W. B. Kibble and A. Vilenkin, Phys. Rev. D52 (1995) 679; J. Ahonen and K. Enqvist, Phys.Rev. D57 (1998) 664; T. Vachaspati, Phys. Lett. B265 (1991) 258; O. Tornkvist, FERMILAB-Pub-97/257-A (hep-ph/9707513), Phys. Rev. D (to appear).

[4] K. Enqvist and P. Olesen, Phys. Lett. B319 (1993) 178; M. Turner and L. Widrow, Phys. Rev. D37 (1988) 2743; K. Enqvist, Invited Talk at the Strong and Electroweak Matter '97 (astro-ph/9707300) and HIP-1998-17/ph (astro-ph/9803196).

[5] J. Schwinger, Phys. Rev. 82 (1951) 664.

[6] M. G. Schmidt and C. Schubert, Phys. Lett. B318 (1993) 438; R. Iengo and G. Jug, Phys. Rev. B52 (1995) 7537.

[7] K. Kajantie, M. Laine, K. Rummukainen and M. Schaposnikov, Phys. Rev. Lett. 77 (1996) 2887; W. Buchmuller and O. Philipsen, Nucl. Phys. B443 (1995) 47; W. Buchmuller and Z. Fodor, Phys. Lett. B331 (1994) 124.

[8] M. Dine, R. G. Leigh, P. Huet, A. Linde and D. Linde, Phys. Rev. D46 (1992) 550

[9] A. D. Linde, Nucl. Phys. B216 (1983) 421

[10] A. Guth and E. Weinberg, Phys. Rev. D23 (1981) 876; G. Anderson and L. Hall, Phys. Rev. D45 (1992) 2685.

[11] D. Grasso and H. Rubinstein, Astrop. Phys. 3 (1995) 95; P. J. Kernan, G. D. Starkman and T. Vachaspati, Phys. Rev. D56 (1997) 3766; B. Cheng, A. V. Olinto, D. J. Schramm and J. W. Truran, Phys. Rev. D54 (1996) 4714. 
[12] P. Elmfors, K. Enqvist and K. Kainulainen, hep-ph/9806403; K. Kajantie, M. Laine, J. Peisa, K. Rummukainen and M. Shaposhnikov, hep-lat/9809004.

[13] M. Carena, M. Quiros, A. Riotto, I. Vilja and C. Wagner, Nucl. Phys. B503 (1997) 404; J. M. Cline and K. Kainulainen, Nucl. Phys. B482 (1996) 73; K. Kajantie, M. Laine, K. Rummukainen and M. Schaposnikov, Nucl. Phys. B446 (1996) 189; M. Losada, Phys. Rev. D56 (1997) 2893. 